\title{
Clinical Evidence Supporting Early Treatment Of Patients With Spinal Muscular Atrophy: Current Perspectives
}

This article was published in the following Dove Press journal:

Therapeutics and Clinical Risk Management

\author{
Tamara Dangouloff' \\ Laurent Servais ${ }^{1,2}$ \\ 'Division of Child Neurology, Centre de \\ Références des Maladies \\ Neuromusculaires, Department of \\ Pediatrics, University Hospital Liège \& \\ University of Liège, Liège, Belgium; \\ ${ }^{2}$ MDUK Neuromuscular Center, \\ Department of Paediatrics, University of \\ Oxford, Oxford, UK
}

\begin{abstract}
Recent advances in the treatment of spinal muscular atrophy (SMA) have dramatically altered prognosis. Rather than a rapidly lethal disease, SMA type 1, the most severe form with the earliest onset of SMA, has become a disease in which long-term eventfree survival with the acquisition of important motor milestones is likely. Prognosis for patients with SMA type 2 has shifted from slow and progressive deterioration to long-term stability. Nevertheless, there is a large heterogeneity in terms of clinical response to currently available treatments, ranging from absence of response to impressive improvement. The only factor identified that is predictive of treatment success is the age of the patient at the initiation of treatment, which is closely related to disease duration. The aim of this paper is to review available evidence that support early intervention using currently available treatment approaches.
\end{abstract}

Keywords: spinal muscular atrophy, nusinersen, zolgensma, risdiplam, branaplam, newborn screening

\section{Introduction}

Spinal muscular atrophy (SMA) is an autosomal recessive, inherited genetic disease characterized by degeneration of alpha motor neurons in the spinal cord. ${ }^{1}$ It is the most common genetic cause of child mortality and has long been considered an incurable disease. The incidence is approximately 1 in 10,000 live births. ${ }^{2,3}$ It is linked to a homozygous deletion of the SMN1 gene. Humans have a variable number of copies of a very closely related gene, SMN2; splicing of the SMN2 transcript usually results in an mRNA lacking exon $7 .{ }^{4}$ The severity of the SMA depends largely on the number of copies of SMN2, a lower number of copies being associated with a more severe phenotype, ${ }^{5}$ yet several exceptions and other genetic modifiers have been reported. ${ }^{6}$

Patients first present with a loss of muscle strength that progresses to paralysis, including paralysis of the respiratory muscles. Clinical phenotypes are grouped into five forms depending on the severity of the disease and the age of onset. SMA type 0 occurs in the neonatal period and causes rapid death. SMA type I (SMA1), also known as Werdnig-Hoffman disease, occurs during the first 6 months of life and is associated with death before 2 years of age in most of the cases in absence of supportive care. Spinal muscular atrophy type II (SMA2), also called "intermediate" SMA, occurs slightly later than type I, between the ages of 6 and 18 months, and is characterized
Correspondence: Laurent Servais Douzième de Ligne, I, Liège 4000, Belgium

Tel +33622592243

Fax +3243218328

Email Iservais@chuliege.be 
by the absence of acquisition of autonomous ambulation. Type III (SMA3) or Kugelberg-Welander disease causes symptoms after the age of 18 months, and these patients acquire autonomous ambulation. SMA2 and SMA3 cause severe disability in children and adults at significant social lifelong costs, estimated at \$US 8.4 and 6.4 million, respectively. ${ }^{7}$ SMA2 and SMA3 progress more slowly than SMA1 but are clearly progressive diseases, even in adulthood. ${ }^{8,9}$ SMA type IV (SMA4), also known as the adult form, occurs during the second or third decade of life, and the ability to walk is preserved.

Several innovative drugs have recently been developed that improve or ameliorate symptoms in many patients. ${ }^{10}$ Nusinersen (marketed as Spinraza, Biogen, Cambridge, MA, USA) is an antisense oligonucleotide drug that is injected intrathecally; it modulates splicing of SMN2 to enhance the production of a functional SMN protein. ${ }^{11}$ Efficacy has recently been demonstrated in a double-blind, placebo-controlled study in patients with SMA1 ${ }^{12}$ and in non-ambulant SMA2 and SMA3 patients younger than 10 years. ${ }^{13}$ Nusinersen was approved by the FDA in December 2016 and by the EMA in June 2017. Nusinersen is the first drug approved to treat pediatric and adult patients with SMA. Open-label studies ${ }^{14-17}$ and real-world data ${ }^{18,19}$ have confirmed the safety and efficacy of the treatment in these patient groups as well as in older SMA1 patients, ${ }^{20}$ a population not covered by the Phase III study. An open-label study of patients previously included in the Phase III trials and a study in pre-symptomatic patients are currently ongoing (NURTURE: NCT02386553; SHINE: NCT02594124).

Other treatments for SMA of certain types are approved or under development. Zolgensma, previously known as zolgensma (Onasemnogene abeparvovec-xioi, Avxs-101, Avexis, Novartis, Bannockburn, IL, USA), ${ }^{21,22}$ is an AAV9-based gene therapy that was approved by the FDA in May 2019 for the treatment of patients younger than 2 years of age. Zolgensma is given as a one-time intravenous administration. It delivers a copy of $S M N$ in a self-complementary adeno-associated viral serotype 9 (scAAV9). ${ }^{23}$ It is under review by the EMA. Large studies are ongoing in SMA1 patients (STR1VE: NCT03306277; STR1VE EU: NCT03461289), and in pre-symptomatic patients (SPR1NT: NCT03505099), Phase I study evaluating an intrathecal approach in SMA2 patients younger than 6 years is also ongoing (STRONG: NCT03381729).

Risdiplam (F. Hoffmann-La Roche Ltd., Basel, Switzerland) and branaplam (Novartis, Basel, Switzerland) are two compounds given orally that modify SMN2 splicing to enhance the production of SMN ${ }^{24}$ Risdiplam is currently in Phase III testing in SMA1 patients (FIREFISH: NCT02913482) and in patients with SMA2 (SUNFISH: NCT02908685) and a pre-symptomatic trial is starting (Rainbowfish: NCT03779334). Phase II testing of Branaplam is ongoing in SMA1 patients (NCT02268552).

Some groups of patients have not been covered by these studies including SMA3 patients and adults with SMA2. In these patients, intrathecal administration, the route used for administration of nusinersen, can be challenging because of scoliosis and/or spinal fusion. Thus, the questions of the potential benefit of treatment, the ratio of benefit to risk, and, importantly, the cost-effectiveness of the treatment remains for several groups of patients. In animals, the importance of early treatment has been extensively demonstrated. ${ }^{25}$ In humans, the main a priori predictive factor of treatment efficacy identified so far in the published studies is the age of patients at treatment initiation, which is closely related to disease duration since most patients for which data are available to have symptom onset between 0 and 18 months. Recently, baseline patients' motor condition was also recognized as a potential predictive factor in zolgensma ${ }^{26}$ and in nusinersen. ${ }^{27}$ The aim of this paper is to review the available evidence that early, pre-symptomatic, treatment of SMA is optimal. Since the data discussed here were acquired in different studies with different study designs with different patient number and characteristics, we chose to present only descriptive statistics but not to report on or to calculate comparative statistics. The various studies and data used are summarized in the following Table 1.

\section{Evidence Supporting Early Treatment With Nusinersen}

\section{Nusinersen In SMAI}

In a double-blind, placebo-controlled, multicenter study conducted in 121 patients with confirmed SMA1 aged between 30 days and 262 days old, two-thirds $(n=80)$ of the children received nusinersen and one-third $(n=41)$ were sham treated. ${ }^{12}$ Of the patients who received nusinersen, 39 had a disease duration of less than 13.1 weeks, and $41 \mathrm{had}$ a disease duration of over 13.1 weeks. Of the 39 patients treated early (ie, those with disease duration less than 13.1 weeks), 30 (77\%) were not on permanentassisted ventilation at the end of the trial, whereas only 19 of $41(46 \%)$ in the latter group were not on permanent ventilation. The difference between untreated and treated patients for time to death or to permanent ventilation was 


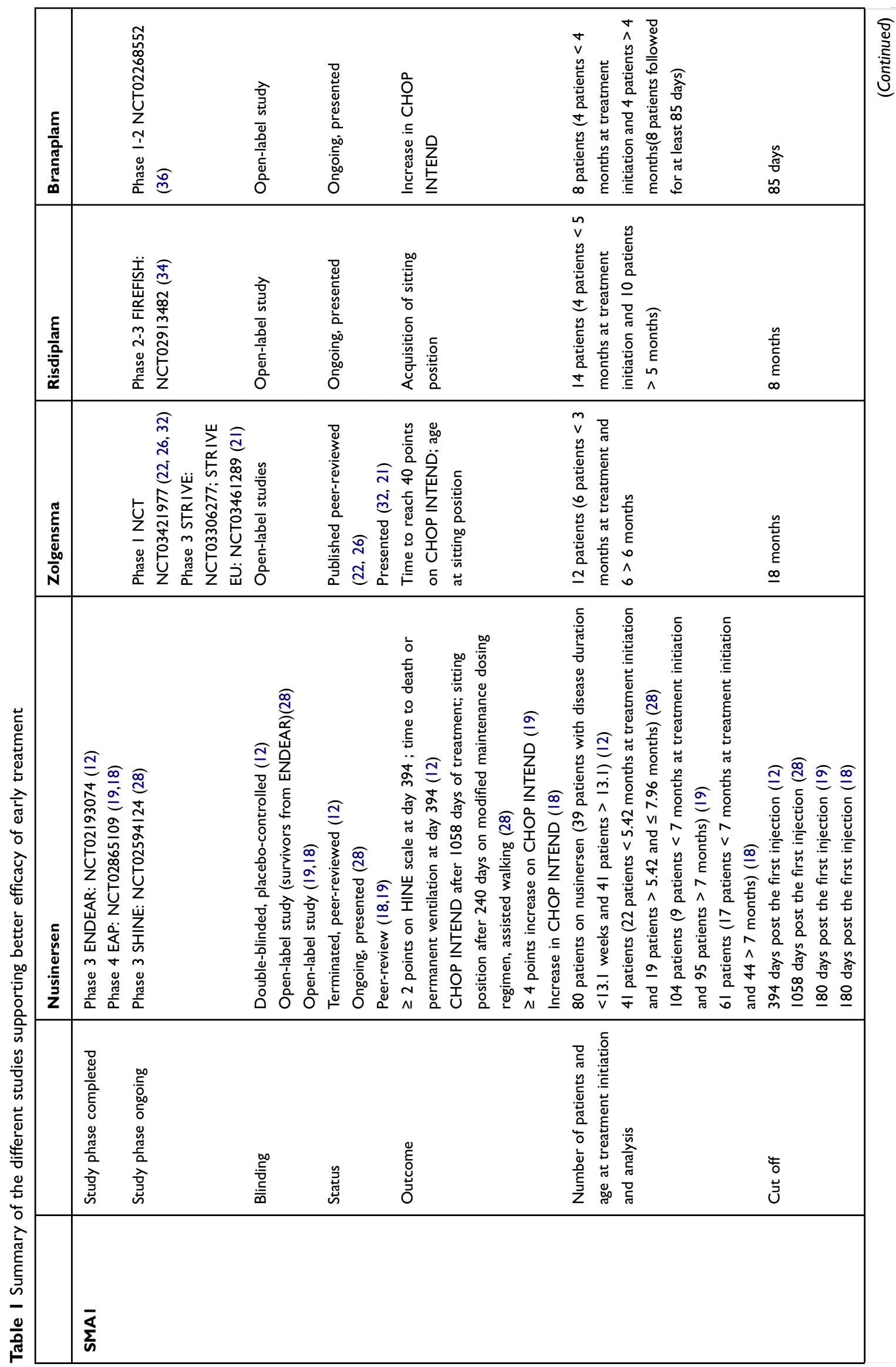




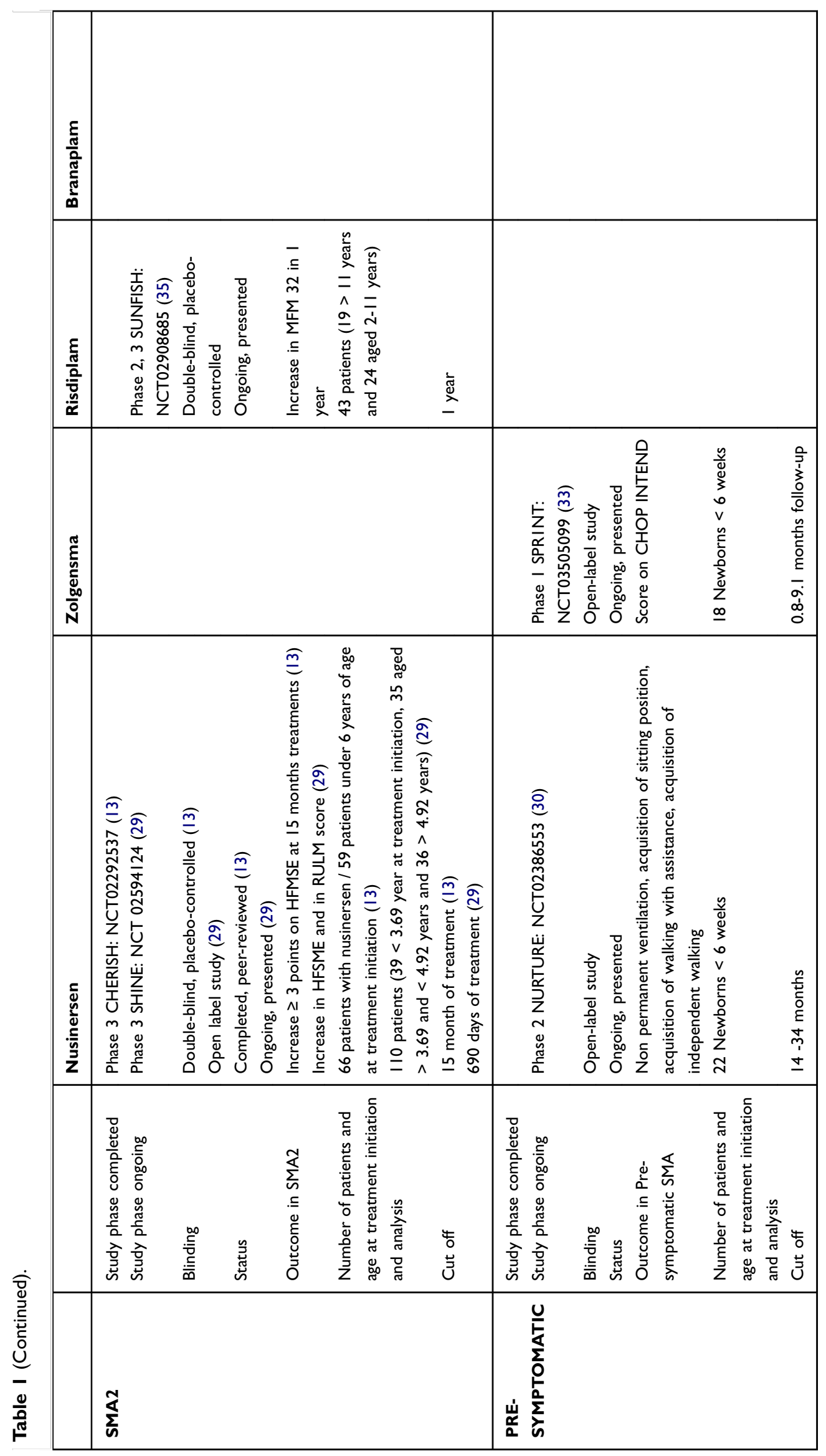


statistically significant only in patients treated early (HR of $0.24)$. The patients treated early also presented with better motor outcomes, since 27 of $29(93 \%)$ of the patients treated early who survived to the end of the study had an increase in HINE-2 score at last visit compared to pretreatment levels. Only 13 of the 29 (45\%) surviving patients in the group that began treatment more than 13.1 weeks after the onset of symptoms had improvements in motor skills.

The survivors were followed in an open-label study. ${ }^{28}$ The 22 children younger than 5.42 months of age at the time of the first injection improved by an average of 19.4 their CHOP INTEND scores (14.8 to 23.9) at 1058 days post the first treatment, whereas the 19 who were between 5.42 months and 7.96 months of age at the time of their first injection improved by 13.8 points (9.2 to 18.4). Similarly, an unsupported sitting position was attained 240 days after modified maintenance dosing regimen was achieved in 18 of $30(60 \%)$ of children treated before 5.42 years but in only 8 of $21(38 \%)$ of those treated later. Three of the $30(10 \%)$ treated before 5.42 months were able to walk with assistance, but none of those treated later were able to walk with assistance.

As part of the Early Access Program (NCT02865109), different teams have continued to observe the effectiveness of nusinersen in a broad cohort of children with SMA1. Of 104 SMA1 patients treated over 6 months in Italy, ${ }^{19}$ seven of the nine patients younger than 7 months at treatment initiation presented with an improvement of more than 4 points $(77 \%)$ on the CHOP INTEND motor evaluation scale, whereas of the 95 patients over 7 months of age at treatment initiation, only 37 (38.94\%) had an improvement of more than 4 points. Similar conclusions were drawn from observation of 61 SMA1 patients treated with nusinersen in Germany. ${ }^{18}$ The 17 patients younger than 7 months at treatment initiation had an average of $14.4 \pm$ 9.2 points improvement in CHOP INTEND score compared to $7.0 \pm 6.6$ points for the 44 in whom treatment was initiated after the age of 7 months.

\section{Nusinersen In SMA2}

A double-blind, placebo-controlled study of nusinersen effectiveness was conducted in 126 patients with SMA2. These patients were aged 2 to 12 years at the time of treatment, had symptoms that appeared after 6 months of age, were able to sit without assistance, had no history of independent walking, and had Hammersmith Functional Motor Scale-Expanded (HFMSE) scores of 10 to $54 .{ }^{13}$ Among these patients, 100 completed the 15-month treatment regimen with 66 allocated to the nusinersen arm and 34 to the sham-treatment arm. Of the 66 patients treated, seven were over 6 years of age and 59 were under 6 years. Only one patient (14\%) older than 6 years of age at baseline was considered as a responder based on improvement by 3 points or more on the HFMSE, whereas 38 of the 59 patients $(64 \%)$ under 6 years of age at treatment initiation improved by 3 points or more.

The duration of the disease also significantly impacted motor progress: in the group of children treated with nusinersen who had disease duration of less than 25 months, 18 of $20(90 \%)$ progressed by 3 points or more, whereas of the 26 first treated when disease duration was between 25 and 44 months only $16(61 \%)$ had a score improvement of 3 points or more. Of the 20 treated after 44 months of disease, only five $(25 \%)$ were considered responders.

Patients in the nusinersen-treated cohort were included in an open-label extension study. ${ }^{29}$ The youngest patients (under 3.69 years of age at their first injection, $n=39$ ) had an average HFMSE scale improvement of $8.6 \pm 0.89$ points after 690 days of treatment, compared to an improvement of $3.0 \pm 0.68$ points for those aged 3.69 to 4.92 years of age at first treatment $(\mathrm{n}=35)$. Those treated at over 4.92 years of age $(n=36)$ lost an average of $2.0 \pm 0.71$ points. The same pattern of improvement according to age at treatment initiation was observed when patients were evaluated based on the Revised Upper Limb Module score, which is used to evaluate the motor performance in the upper limbs for individuals with SMA. Patients younger than 3.69 years had an average improvement of $7.9 \pm 0.78$ points, those in the intermediate group had an average improvement of $3.4 \pm 0.54$, and no change $(+0.6 \pm 0.49)$ was observed for patients older than 4.92 years at treatment initiation.

\section{Nusinersen In Pre-Symptomatic Patients}

NURTURE is an open-label study designed to evaluate the safety and efficacy of nusinersen administered to presymptomatic patients. Twenty-five newborns with two $(\mathrm{n}=15)$ or three $(\mathrm{n}=10)$ copies of $S M N 2$ were included and received the treatment before 6 weeks of age. ${ }^{30}$ At the time of the interim analysis (May 2018), the median (range) age at last visit was 26.0 (14.0-34.3) months. All the children were alive, and none needed permanent ventilation. All (100\%) had reached the sitting position milestone, 22 of $25(88 \%)$ could walk with assistance, and 17 of 22 patients aged more than 18 months $(77 \%)$ could walk independently. This is an outcome dramatically different from patients treated post-symptomatically, ${ }^{28}$ in 
whom $60 \%$ of patients sit independently and $10 \%$ walk with assistance in the group treated early $(<5.5$ months of age at treatment onset) at 8 months after the modified maintenance dosing regimen was initiated. An anecdotal case of a pre-symptomatically treated patient climbing stairs has also been reported. ${ }^{31}$

\section{Data Supporting Early Treatment With Zolgensma}

During a Phase I/II study to evaluate the safety and efficacy of zolgensma, 15 children with SMA1 received a single intravenous injection of the gene therapy agent. ${ }^{26,32}$ All patients are alive at approximately 2 years after treatment, and none required permanent mechanical ventilation. The six patients who received treatment before the age of 3 months reached a CHOP INTEND score of more than 40 more rapidly (median of 11.9 months) than the six patients who received treatment after the age of 3 months (median of 22.2 months). ${ }^{21,22}$ Patients treated before the age of 3 months acquired the sitting position ( $>5 \mathrm{~s}$ ) at a mean age of 9.4 months for those with a high motor function baseline $(n=3)$ and at 17 months $(n=3)$ for those with a low motor function at baseline. In contrast, patients treated after the age of 3 months $(n=6)$ reached an unassisted sitting position at the median age of 22 months. ${ }^{26}$

SPR $1 N T^{33}$ is a Phase III study designed to assess the safety and efficacy of zolgensma in pre-symptomatic SMA patients treated before the age of 6 weeks. Eight patients with two copies of SMN2, nine with three copies, and one with four copies were included. In March 2019 (after follow-up ranging from 0.8 to 9.1 months), all patients were alive, and none required permanent ventilation. On the CHOP INTEND scale, of the eight patients with two copies of SMN2, all had obtained a score higher than 50 points, six had scores higher than 60, and three had obtained the maximum score of 64 . Comparing the results of these two different studies - one still ongoing - is challenging, but these results indicate that when SMA patients are treated pre-symptomatically with zolgensma the increase in CHOP INTEND score is more rapid than when patients are treated after symptom onset and that in symptomatic patients treatment before 3 months is more beneficial than treatment after the age of 3 months.

\section{Data Supporting Early Treatment With Risdiplam}

Better efficacy when treatment is initiated early is also suggested in the first results from the FIREFISH (NCT02913482), an open-label two-part trial to evaluate the optimal dose, the safety, and the efficacy of oral treatment of SMA1 patients with risdiplam. In 17 patients with SMA1 treated with the highest dose of risdiplam, six were younger than 5 months, and 11 were older. Fourteen children survived through 8 months of follow-up. Four of the survivors had been included before the age of 5 months and 10 after the age of 5 months. In the earlier treatment group, three of four $(75 \%)$ reached the sitting position (independent and with support at hips), compared to three of ten (30\%) in those treated after 5 months. ${ }^{34}$ In 43 patients with SMA2 and SMA3 treated for 1 year with risdiplam, ${ }^{35}$ the mean improvement on the MFM32 scale was 1.64 points in patients older than 11 years $(n=19)$ and 3.47 points in patients aged $2-11$ years $(n=24)$. The proportion of patients who improved by more than 3 points was $71 \%$ in the younger patients and $42 \%$ in the older.

\section{Data Supporting Early Treatment With Branaplam}

The first results of clinical evaluation of the oral therapy branaplam (NCT02268552) in an open-label, multi-part study in infants with SMA1 having two copies of SMN2 suggested a better improvement in patients included before the age of 4 months than in those included after the age of 4 months. ${ }^{36}$ Twenty-five patients with onset of symptoms before 6 months of age and less than 180 days of age at screening were included in this study. As of April 10, 2019 , with a median follow-up of 2.2 months (0.3-10 months), the mean CHOP INTEND increase in the eight patients followed for at least 85 days of treatment was 6.0 in four patients younger than 4 months at inclusion and 3.5 in four patients older than 4 months.

\section{Discussion}

There is a concordant set of data from different trials in SMA1 and SMA2 patients that indicate that efficacy of treatment is enhanced when patients are treated soon after or before symptom onset. Some of these data were acquired during completed double-blind, randomized, placebo-controlled studies, others were collected during open-label studies and very preliminary. Some of the data reviewed here are published in peer-reviewed journals and some have been publically presented and are available only as abstracts. Thus, the strength of the data described here is very heterogeneous. Nevertheless, all the data suggest that efficacy is optimal when treatment is initiated before or soon after the onset of symptoms. We chose to 
discuss here not only peer-reviewed, but also non-peerreviewed data since meeting presentations are mostly confined to a very specialized audience, but decision-making on newborn screening or treatment of patients does not rely only on the limited circle of clinical trial investigators. Our goal with this review is to ensure that policy makers and physicians are aware of the body of evidence - which is publically available although not necessarily peerreviewed - that indicates the value of early treatment of SMA1 and SMA2 patients. The data presented here consider early treatment as the time of instauration of a disease-modifying drug. Nevertheless, all data were acquired in patients who were also treated with standard of care, which was an inclusion criterion in all studies. Therefore, early treatment should be also understood as early management, including multidisciplinary care follow-up.

Achieving a better treatment response is important not only from the patient perspective but also from an economic point of view. Although the costs of these treatments are very high, so is the social cost of the disease. The cost of treatment with nusinersen is $\$ 558,000$ in the first year (six doses) and $\$ 279,000$ per year for maintenance treatment (three doses). ${ }^{37}$ The announced price of zolgensma is $\$ 2.125$ million for the single injection. ${ }^{38}$ In comparison, however, the estimated lifelong cost of medical support for SMA2 and SMA3 patients has been estimated at $\$ 8.4$ and $\$ 6.4$ million, respectively. ${ }^{7}$

The cost of untreated SMA1 ( $\$ 120,000$ per year) is much lower than that for medical support for SMA2 and SMA3 patients. Patients with SMA1 currently have a life expectancy of only 2 years. By increasing the life expectancy of these patients, the cost related to their disability will dramatically increase resulting in a negative cost-to-effectiveness evaluation. For example, nusinersen treatment provides overall benefits in terms of adjusted survival and quality of life per year, both for patients and their caregivers. ${ }^{39}$ However, with additional costs in excess of $\$ 2.4$ million, ${ }^{39}$ mainly associated with maintenance treatment over the lifetime of the patient, and frequent hospitalization for respiratory events, ${ }^{40}$ this is not considered a cost-effective threshold, even for a rare disease. Obtaining a much better efficacy from the same drug by initiating the treatment earlier has the potential to improve this ratio sufficiently to make treatment worthwhile.

Pre-symptomatic studies conducted with zolgensma and nusinersen indicate an efficacy far above than observed in post-symptomatic patients. ${ }^{30,33}$ Although longer follow-up is needed to confirm that this short-term efficacy translates into a significant long-term benefit for the patient, it appears that early diagnosis should be facilitated. A meta-analysis published in $2015^{41}$ showed that the time between symptom onset to diagnosis was considerable: the weighted mean ages of confirmed SMA genetic diagnoses were 6.3, 20.7, and 50.3 months in SMA1, 2, and 3 patients, respectively. It is likely that physician and caregiver awareness related to media attention surrounding the innovative medications now available to treat SMA will decrease time to diagnosis. Nevertheless, given the rapid evolution of the disease, especially in SMA1 patients, even reducing the diagnosis delay by $50 \%$ will not allow treatment of more than $50 \%$ of patients below the age of 3 months.

Neonatal screening would expedite diagnosis and ensure early treatment. Two pilot programs in Taiwan ${ }^{42}$ and New York ${ }^{43}$ validated the feasibility of SMA newborn screening (NBS). NBS for SMA is now performed in Taiwan and is registered in the Recommended Uniform Screening Panel (RUSP) in the USA. It is recommended in many states in the USA but is not mandatory. Extended pilot programs started in early 2018 in Germany and Belgium $^{44,45}$ and in early 2019 in Italy. Similar programs are planned to start elsewhere in Europe in the coming years. $^{46}$

\section{Conclusion}

Innovative therapeutic approaches for the treatment of spinal muscular atrophy have altered the prognosis for patients with this generally fatal disease. Independently of the mode of action, emerging data suggest that the treatments discussed here have better efficacies when patients are treated pre-symptomatically or soon after symptoms are observed rather than months after symptom onset. In the context of ultra-expensive medication and burdensome disease leading to severe disability in patients treated late, newborn screening is arguably the best solution to optimize the effect of the innovative therapies that are changing the prognosis for patients with spinal muscular atrophy.

\section{Acknowledgments}

We thank Jacqueline R. Wyatt for editing the manuscript.

\section{Disclosure}

TD received honorarium for lectures from Biogen. LS is principal investigator in Biogen-, Roche-, and Avexis-sponsored studies; serves on scientific advisory boards of Biogen, Roche, Avexis, and the SMA foundation; and received 
honorarium for lectures and consultancies from Roche and Biogen. The authors report no other conflicts of interest in this work.

\section{References}

1. Kolb SJ, Kissel JT. Spinal muscular atrophy. Neurol Clin. 2015;33 (4):831-46. doi:10.1016/j.ncl.2015.07.004

2. Pearn J. Incidence, prevalence, and gene frequency studies of chronic childhood spinal muscular atrophy. J Medical Genet. 1978;15 (6):409-413. doi:10.1136/jmg.15.6.409

3. Prior TW, Snyder PJ, Rink BD, et al. Newborn and carrier screening for spinal muscular atrophy. Am J Med Genet A. 2010;152a(7):16081616. doi:10.1002/ajmg.a.33474

4. Lefebvre S, Burglen L, Reboullet S, et al. Identification and characterization of a spinal muscular atrophy-determining gene. Cell. 1995;80(1):155-165. doi:10.1016/0092-8674(95)90460-3

5. Calucho M, Bernal S, Alias L, et al. Correlation between SMA type and SMN2 copy number revisited: an analysis of 625 unrelated Spanish patients and a compilation of 2834 reported cases. Neuromuscul Disord. 2018;28(3):208-215. doi:10.1016/j.nmd.2018. 01.003

6. Wirth B, Garbes L, Riessland M. How genetic modifiers influence the phenotype of spinal muscular atrophy and suggest future therapeutic approaches. Curr Opin Genet Dev. 2013;23(3):330-338. doi:10.1016/j. gde. 2013.03 .003

7. Klug C, Schreiber-Katz O, Thiele S, et al. Disease burden of spinal muscular atrophy in Germany. Orphanet $J$ Rare Dis. 2016;11(1):58. doi:10.1186/s13023-016-0424-0

8. Chabanon A, Seferian AM, Daron A, et al. Prospective and longitudinal natural history study of patients with type 2 and 3 spinal muscular atrophy: baseline data NatHis-SMA study. PLoS One. 2018;13(7):e0201004. doi:10.1371/journal.pone.0201004

9. Mazzone E, Bianco F, Main M, et al. Six minute walk test in type III spinal muscular atrophy: a 12 month longitudinal study. Neuromuscul Disord. 2013;23(8):624-628. doi:10.1016/j.nmd.2013.06.001

10. Daron A, Delstanche S, Dangouloff T, Servais L. [Infantile spinal muscular atrophy: therapeutic (R)evolution]. Rev Med Liege. 2019;74(2):8285.

11. Hua Y, Sahashi K, Hung G, et al. Antisense correction of SMN2 splicing in the CNS rescues necrosis in a type III SMA mouse model. Genes Dev. 2010;24(15):1634-1644. doi:10.1101/gad.1941310

12. Finkel RS, Mercuri E, Darras BT, et al. Nusinersen versus sham control in infantile-onset spinal muscular atrophy. $N$ Engl $J M$. 2017;377(18):1723-1732. doi:10.1056/NEJMoa1702752

13. Mercuri E, Darras BT, Chiriboga CA, et al. Nusinersen versus sham control in later-onset spinal muscular atrophy. N Engl J M. 2018;378 (7):625-635. doi:10.1056/NEJMoa1710504

14. Finkel RS, Chiriboga CA, Vajsar J, et al. Treatment of infantile-onset spinal muscular atrophy with nusinersen: a phase 2, open-label, doseescalation study. Lancet. 2016;388(10063):3017-3026. doi:10.1016/ S0140-6736(16)31408-8

15. Hache M, Swoboda KJ, Sethna N, et al. Intrathecal injections in children with spinal muscular atrophy: nusinersen clinical trial experience. J Child Neurol. 2016;31(7):899-906. doi:10.1177/088 3073815627882

16. Chiriboga CA, Swoboda KJ, Darras BT, et al. Results from a phase 1 study of nusinersen (ISIS-SMN(Rx)) in children with spinal muscular atrophy. Neurology. 2016;86(10):890-897. doi:10.1212/WNL.000000 0000002445

17. Darras BT, Chiriboga CA, Iannaccone ST, et al. Nusinersen in lateronset spinal muscular atrophy: long-term results from the phase $1 / 2$ studies. Neurology. 2019;92(21):e2492-e2506. doi:10.1212/WNL.00 00000000007527
18. Pechmann A, Langer T, Schorling D, et al. Evaluation of children with SMA type 1 under treatment with nusinersen within the expanded access program in Germany. J Neuromuscul Dis. 2018;5 (2):135-143. doi:10.3233/JND-180315

19. Pane M, Coratti G, Sansone VA, et al. Nusinersen in type 1 spinal muscular atrophy: 12-month real world data. Ann Neurol. 2019. Epub 2019 Jul 8. doi:10.1002/ana.25533

20. Aragon-Gawinska K, Seferian AM, Daron A, et al. Nusinersen in patients older than 7 months with spinal muscular atrophy type 1: a cohort study. Neurology. 2018;91(14):e1312-e1318. doi:10.1212/ WNL.0000000000005773

21. Mendell JR, Al-Zaidy S, Shell R, et al. Single-dose gene-replacement therapy for spinal muscular atrophy. $N$ Engl J Med. 2017;377 (18):1713-1722. doi:10.1056/NEJMoa1706198

22. Lowes L, Al-Zaidy S, Shell R, et al. AVXS-101 phase 1 gene therapy clinical trial in SMA type 1: patients treated early with the proposed therapeutic dose were able to sit unassisted at a younger age. Neuromuscul Disord. 2017;27:S208-S209. doi:10.1016/j.nmd.2017.06. 414

23. Avexis. What is ZOLGENSMA? Published 2019. [Updated June, 2019]. Available from: https://www.zolgensma.com/what-is-zolgen sma. Accessed July 26, 2019.

24. Naryshkin NA, Weetall M, Dakka A, et al. Motor neuron disease. SMN2 splicing modifiers improve motor function and longevity in mice with spinal muscular atrophy. Science (New York, NY). 2014;345(6197):688-693. doi:10.1126/science.1250127

25. Sleigh JN, Gillingwater TH, Talbot K. The contribution of mouse models to understanding the pathogenesis of spinal muscular atrophy. Dis Model Mech. 2011;4(4):457. doi:10.1242/dmm.007245

26. Lowes LP, Alfano LN, Arnold WD, et al. Impact of age and motor function in a phase $1 / 2 \mathrm{~A}$ study of infants with SMA type 1 receiving single-dose gene replacement therapy. Pediatr Neurol. 2019. Epub 2019 May 13. doi:10.1016/j.pediatrneurol.2019.05.005

27. Aragon-Gawinska K, Daron A, Ulinici A, et al. Sitting in nusinersentreated spinal muscular atrophy type 1 patients. Dev Med Child Neurol. In revision.

28. Finkel R, Castro D, Farrar M, et al. Interim report on the safety and efficacy of longer-term treatment with nusinersen in infantile-onset Spinal Muscular Atrophy (SMA): updated results from the SHINE study. Communication presented at American Academy of Neurology 2019 - 71st Annual Meeting; May 4-10, 2019; Philadelphia, PA, USA.

29. Darras B, Farrar M, Mercuri E, et al. Interim report on the safety and efficacy of longer-term treatment with nusinersen in later-onset Spinal Muscular Atrophy (SMA): results from the SHINE study. Communication presented at American Academy of Neurology 2019-71st Annual Meeting; May 4-10, 2019; Philadelphia, PA, USA.

30. De Vivo DC, Topaloglu H, Swoboda KJ, et al. Nusinersen in infants who initiate treatment in a presymptomatic stage of Spinal Muscular Atrophy (SMA): interim efficacy and safety results from the phase 2 NURTURE study (S25.001). Communication presented at American Academy of Neurology 2019-71st Annual Meeting; May 4-10, 2019; Philadelphia, PA, USA.

31. Gidaro T, Servais L. Nusinersen treatment of spinal muscular atrophy: current knowledge and existing gaps. Dev Med Child Neurol. 2019;61(1):19-24. doi:10.1111/dmcn.14027

32. Mendell J, Lehman K, McColly M, et al. AVXS-101 GeneReplacement Therapy (GRT) in Spinal Muscular Atrophy type 1 (SMA1): long-term follow-up from the phase 1 clinical trial (S25.006). Communication presented at American Academy of Neurology 2019-71st Annual Meeting; May 4-10, 2019; Philadelphia, PA, USA. doi:10.1017/cjn.2019.166

33. Strauss KA, Swoboda KJ, Farrar M, et al. AVXS-101 gene-replacement therapy in presymptomatic spinal muscular atrophy: SPR1NT study update. Communication presented at American Academy of Neurology 2019-71st Annual Meeting; May 4-10, 2019; Philadelphia, PA, USA. 
34. Seabrook T, Baranello G, Servais L, et al. FIREFISH part 1: early clinical results following an increase ofSMN protein in infants with type 1 spinal muscular atrophy (SMA) treated with risdiplam (RG7916). Communication presented at MDA Clinical \& Scientific Conference; April 13-17, 2019; Orlando, Florida.

35. Mercuri E, Baranello G, Kirschner J, et al. Update from SUNFISH part 1: safety, tolerability and PK/PD from the dose-finding study, including exploratory efficacy data, in patients with type 2 or 3 spinal muscular atrophy (SMA) treated with risdiplam (RG7916) Communication presented at American Academy of Neurology 2019-71st Annual Meeting; May 4-10, 2019 Philadelphia, PA, USA

36. Jevtic S, Carr D, Dobrzycka-Ambrozevicz A, et al. Branaplam in type 1 spinal muscular atrophy: second part of a phase I/II study. Communication presented at 23rd SMA researcher meeting, Cure SMA; June 28-30, 2019; Disneyland hotel in Anaheim, California.

37. CADTH Common Drug Reviews. Pharmacoeconomic Review Report: Nusinersen (spinraza): (biogen Canada Inc.): Indication: Treatment of Patients with $5 q$ SMA. Ottawa (ON): Canadian Agency for Drugs and Technologies in Health; 2018.

38. Relations NM AveXis Announces Innovative Zolgensma ${ }^{\circledR}$ Gene Therapy Access Programs for US Payers and Families. Published 2019, May 24. Available from: https://www.novartis.com/news/mediareleases/avexis-announces-innovative-zolgensma-gene-therapy-accessprograms-us-payers-and-families. Accessed July 26, 2019.

39. Zuluaga-Sanchez S, Teynor M, Knight C, et al. Cost effectiveness of nusinersen in the treatment of patients with infantile-onset and lateronset spinal muscular atrophy in Sweden. PharmacoEconomics. 2019;37(6):845-865. doi:10.1007/s40273-019-00769-6
40. Ali I, Gilchrist F, Carroll WD, et al. G176(P) healthcare utilisation in SMA type 1 patients treated with nusinersen. Arch Disease Child. 2019;104(Suppl 2):A71.

41. Lin CW, Kalb SJ, Yeh WS. Delay in diagnosis of spinal muscular atrophy: a systematic literature review. Pediatr Neurol. 2015;53 (4):293-300. doi:10.1016/j.pediatrneurol.2015.06.002

42. Chien YH, Chiang SC, Weng WC, et al. Presymptomatic diagnosis of spinal muscular atrophy through newborn screening. J Pediatr. 2017;190:124-129.e121. doi:10.1016/j.jpeds.2017.0 6.042

43. Kraszewski JN, Kay DM, Stevens CF, et al. Pilot study of population-based newborn screening for spinal muscular atrophy in New York state. Genet Med. 2018;20(6):608-613. doi:10.1038/ $\operatorname{gim} .2017 .152$

44. Boemer F, Caberg JH, Dideberg V, et al. Newborn screening for SMA in Southern Belgium. Neuromuscul Disord. 2019;29(5):343349. doi:10.1016/j.nmd.2019.02.003

45. Boemer F, Caberg J, Dideberg V, et al. (S)un (M)ay (A)rise on SMA: l'espoir d'une région sans amyotrophie spinale [(S)un (M) ay (A)rise on SMA: the hope of a region without spinal muscular atrophy]. Rev Med Liege. 2019;74(9):461-464. In Press.

46. Dangouloff T, Burghes A, Bertini E, et al. 244th ENMC international workshop: newborn screening in spinal muscular atrophy,10th-12th May 2019, Hoofdorp, The Netherlands. Neuromuscul Disord. Submitted.
Therapeutics and Clinical Risk Management

\section{Publish your work in this journal}

Therapeutics and Clinical Risk Management is an international, peerreviewed journal of clinical therapeutics and risk management, focusing on concise rapid reporting of clinical studies in all therapeutic areas, outcomes, safety, and programs for the effective, safe, and sustained use of medicines. This journal is indexed on PubMed Central, CAS,

\section{Dovepress}

EMBase, Scopus and the Elsevier Bibliographic databases. The manuscript management system is completely online and includes a very quick and fair peer-review system, which is all easy to use. Visit http://www.dovepress.com/testimonials.php to read real quotes from published authors. 